\title{
WEIERSTRASS DIVISION WITH QUASIANALYTIC BOUNDARY VALUES
}

\section{L. CHILDRESS}

\section{Introduction}

Let $g: \mathbf{R}^{+} \rightarrow \mathbf{R}^{+} \quad\left(\mathbf{R}^{+}\right.$is the set of nonnegative real numbers) be a convex increasing function such that $g(0)=0$ and $t^{-1} g(t) \rightarrow+\infty$ as $t \rightarrow+\infty$. Define a sequence $\left\{M_{n}\right\}_{n \in Z^{+}}\left(\mathbb{Z}^{+}\right.$is the set of nonnegative integers) by $M_{n}=\exp g(n), n \in \mathbb{Z}^{+}$. We assume $g$ grows fast enough to ensure that $M_{n} \geqq n !, n \in \mathbb{Z}^{+}$.

Let $\Omega$ be a domain in $\mathbb{C}^{k}$ with 0 a point on its boundary $\mathrm{b} \Omega$. We denote by $\mathscr{A}_{k}={ }_{0} \mathscr{A}_{k}\left(\left\{M_{n}\right\}, \Omega\right)$ the set of germs at 0 of complex-valued Whitney $C^{\infty}$ functions $f$ on $\bar{\Omega}$ (the closure of $\Omega$ ) which are analytic in $\Omega$ and satisfy the following growth conditions on their derivatives: for each $r>0$ sufficiently small that $f$ is represented by a function on $\bar{\Omega} \cap \Delta_{k}(r)\left(\Delta_{k}(r)=\left\{z \in \mathbf{C}^{k}:\left|z_{j}\right|<r, 1 \leqq j \leqq \mathrm{k}\right\}\right)$ there exist constants $A$ and $B$, which depend in general on both $f$ and $r$ but not on $n \in \mathbb{Z}^{+}$, such that for all $n \in \mathbb{Z}^{+}$,

$$
\sup _{\substack{\alpha \in\left(\mathrm{Z}^{+}\right)^{k},|\alpha| \leqq n, z \in \bar{\Omega} \cap \boldsymbol{A}_{k}(r)}}\left|D^{\alpha} f(z)\right| \leqq A B^{n} M_{n} .
$$

$\left(D^{\alpha}=D_{z}^{\alpha}=\partial^{|\alpha|} / \partial z_{1}^{\alpha_{1}} \ldots \partial z_{k}^{\alpha_{k}}\right.$, where $z=\left(z^{\prime}, z_{k}\right)=\left(z_{1}, \ldots, z_{k}\right)$ are coordinates on $\mathrm{C}^{k}$, $\alpha=\left(\alpha_{1}, \ldots, \alpha_{k}\right) \in\left(\mathbf{Z}^{+}\right)^{k}$, and $\left.|\alpha|=\alpha_{1}+\ldots+\alpha_{k}.\right)$ We assume that $\mathscr{A}_{k}$ is quasianalytic in the sense of Denjoy and Carleman:

$$
f \in \mathscr{A}_{k} \text { and } D^{\alpha} f(0)=0 \text { for all } \quad \alpha \in\left(\mathbb{Z}^{+}\right)^{k} \text { imply } f=0 \in \mathscr{A}_{k} .
$$

Before going on, we remark that by the use of the logarithmic convexity of the sequence $\left\{M_{n}\right\}$, it is not difficult to show that $\mathscr{A}_{k}$ is a local algebra with maximal ideal $m_{k}=\left\{f \in \mathscr{A}_{k}: f(0)=0\right\}$. The quasianalyticity assumption is independent of the dimension $k$. If the sequence $\left\{M_{n}\right\}$ satisfies certain additional hypotheses, then $\mathscr{A}_{k}$ is closed under composition whenever the composition makes sense, and $\mathscr{A}_{k}$ is also closed under differentiation. For a more complete discussion, see [2].

In this paper we consider a quasianalytic local algebra $\mathscr{A}_{k}\left(\left\{M_{n}\right\}\right)$. We show a Weierstrass-Malgrange-Mather type division theorem does not hold in $\mathscr{A}_{k}\left(\left\{M_{n}\right\}\right)$ 
if $k \geqq 2, \mathscr{A}_{k}(\{n !\})$ is a proper subset of $\mathscr{A}_{k}\left(\left\{M_{n}\right\}\right)$, and $\mathrm{b} \Omega$ is $C^{2}$ smooth and strongly Levi pseudoconvex at 0 . If, however, $b \Omega$ is Levi pseudoflat at 0 , we prove a generic division theorem holds in $\mathscr{A}_{k}\left(\left\{M_{n}\right\}\right), k \geqq 2$. We further show in this case that division is possible in $\mathscr{A}_{k}\left(\left\{M_{n}\right\}\right)$ by every regular element of $\mathcal{O}_{k}$, the local algebra of germs at 0 of analytic functions. (The case in which $\mathrm{b} \Omega$ is pseudoconcave at 0 is trivial, since in this case $\mathscr{A}_{k}\left(\left\{M_{n}\right\}\right)$ reduces to $\mathcal{O}_{k}$. See L. Hörmander, [4].)

\section{Preliminaries}

The following proposition is any easy consequence of the closed graph theorem:

Proposition 2.1. Let $E$ be a Banach space and $F=\cup_{n=1}^{+\infty} F_{n}$ be an inductive limit of Banach spaces. If $T: E \rightarrow F$ is a continuous linear map, then there exists a positive integer $n_{0}$ such that $T(E) \subseteq F_{n_{0}}$.

We will apply this proposition to estimate the derivatives of the quotient and remainder when we divide by a fixed regular element $f \in \mathscr{A}_{k}\left(\left\{M_{n}\right\}\right)$. The result will be that the growth of the derivatives of the element we are dividing by $f$ determines the growth of the derivatives of the quotient and remainder.

For positive integers $v$ and $N$, let

$$
\begin{aligned}
& A_{k, v, N}=\left\{f: f \text { is a Whitney } C^{\infty} \text { function on } \bar{\Omega} \cap \Delta_{k}(1 / v)\right. \\
& \left.\quad \text { and } \sup _{n \in \mathbf{Z}^{+}} \sup _{\substack{\alpha \in\left(\mathbf{Z}^{+}\right)^{k},|\alpha| \leqq n, z \in \bar{\Omega} \cap \Delta_{k}(1 / v)}}\left|D^{\alpha} f(z)\right| / N^{n} M_{n}<+\infty\right\} .
\end{aligned}
$$

Note that for all positive integers $v$ and $N, A_{k, v, N}$ is a Banach space, and the inductive limit $\bigcup_{v, N=1}^{+\infty} A_{k, v, N}$ may be identified with $\mathscr{A}_{k}\left(\left\{M_{n}\right\}\right)$.

Fix $f \in \mathscr{A}_{k}\left(\left\{M_{n}\right\}\right)$, which is regular in $z_{k}$ of order $d$. (This means $f(0)=\partial f(0) / \partial z_{k}=$ $=\ldots=\partial^{d-1} f(0) / \partial z_{k}^{d-1}=0$, while $\partial^{d} f(0) / \partial z_{k}^{d} \neq 0$.) Let $v_{0}$ be the smallest positive integer such that $f$ is represented by a function on $\Delta_{k}(1 / v)$ for all $v \geqq v_{0}$. We define a map

$$
\begin{gathered}
\left(q, r_{1}, \ldots, r_{d}\right) \rightarrow g=f q+\sum_{j=1}^{d} r_{j} z_{k}^{d-j}, \\
\bigcup_{\substack{v=v_{0}, N=1}}^{+\infty}\left(A_{k, v, N} \oplus\left(\bigoplus_{1}^{d} A_{k-1, v, N}\right)\right) \rightarrow \bigcup_{\substack{v=v_{0}, N=1}}^{+\infty} A_{k, v, N} .
\end{gathered}
$$

This map is continuous, for its restriction to each direct summand $A_{k, v, N} \oplus$ $\left(\oplus_{1}^{d} A_{k-1, v, N}\right)$ is continuous. The assumption that the $\mathscr{A}_{k}\left(\left\{M_{n}\right\}\right)$ are quasianalytic implies the map is injective. The map is surjective if and only if division by $f$ is pos- 
sible within $\mathscr{A}_{k}\left(\left\{M_{n}\right\}\right)$. If the map is surjective, the closed graph theorem implies its inverse is continuous. It is to this inverse map that we apply Proposition 2.1.

Given positive integers $v \geqq v_{0}$ and $N$, Proposition 2.1 implies that there exist positive integers $v^{\prime} \geqq v_{0}$ and $N^{\prime}$, as well as a constant $A$, such that for each $g \in A_{k, v, N}$, there exist $q \in A_{k, v^{\prime}, N^{\prime}}$, and $r_{j} \in A_{k-1, v^{\prime}, N^{\prime}}, 1 \leqq j \leqq d$, which satisfy

$$
g=f q+\sum_{j=1}^{d} r_{j} z_{k}^{d-j}
$$

and which also satisfy the estimates

$1 \leqq j \leqq d$, and

$$
\begin{aligned}
& \sup _{n \in \mathbf{Z}^{+}} \sup _{\substack{\alpha \in\left(\mathbf{Z}^{+}\right)^{k-1},|\alpha| \leqq n, z^{\prime} \in \bar{\Omega} \cap \Delta_{k-1}\left(1 / v^{\prime}\right)}}\left|D^{\alpha} r_{j}\left(z^{\prime}\right)\right| /\left(N^{\prime}\right)^{n} M_{n} \\
& \leqq A \sup _{n \in \mathbb{Z}^{+}} \sup _{\substack{\beta \in\left(\mathbf{Z}^{+}\right)^{k},|\beta| \leqq n, z \in \bar{\Omega} \cap \dot{\Delta}_{k}(1 / v)}}\left|D^{\beta} g(z)\right| / N^{n} M_{n},
\end{aligned}
$$

$$
\begin{aligned}
& \sup _{n \in \mathbf{Z}^{+}} \sup _{\substack{\beta \in\left(\mathbf{Z}^{+}\right)^{k},|\beta| \leqq n, z \in \bar{\Omega} \cap \Delta_{k}\left(1 / v^{\prime}\right)}}\left|D^{\beta} q(z)\right| /\left(N^{\prime}\right)^{n} M_{n} \\
& \leqq A \sup _{n \in \mathbf{Z}^{+}} \sup _{\substack{\beta \in\left(\mathbf{Z}^{+}\right)^{k},|\beta| \leqq n, z \in \bar{\Omega} \cap \Delta_{k}(1 / v)}}\left|D^{\beta} g(z)\right| / N^{n} M_{n} .
\end{aligned}
$$

We summarize these results as a lemma.

Lemma 2.2. Let $f \in \mathscr{A}_{k}\left(\left\{M_{n}\right\}\right)$ be regular in $z_{k}$ of order $d$, and suppose that division by $f$ is possible within $\mathscr{A}_{k}\left(\left\{M_{n}\right\}\right)$. Let $v_{0}$ be the smallest positive integer such that $f$ is represented by a function on $\Delta_{k}(1 / v)$ for all $v \geqq v_{0}$. Given positive integers $v \geqq v_{0}$ and $N$, there exist positive integers $v^{\prime} \geqq v_{0}$ and $N^{\prime}$, as well as a constant $A$, such that for each $g \in A_{k, v, N}$, there exist $q \in A_{k, v^{\prime}, N^{\prime}}$ and $r_{j} \in A_{k-1, v^{\prime}, N^{\prime}}, 1 \leqq j \leqq d$, which satisfy equation (2.1) and estimates (2.2) and (2.3).

We will need one technical lemma, which we now state. The proof may be found in [2].

Lemma 2.3. Let $\lambda(a)=\sup _{n \in Z^{+}}\left|a^{n}\right| M_{n}$ for $a \in \mathbf{C}$, and suppose there exist $\varepsilon>0, A>0$, and $C>0$ such that

$$
\exp (\varepsilon a) \leqq C \lambda(a), \quad a \in \mathbf{R}, \quad a>A .
$$

Then there exist $\alpha>0$ and $\beta>0$ such that

$$
M_{n} \leqq \alpha \beta^{n} n !, \quad n \in \mathbf{Z}^{+} .
$$




\section{The case of a strongly pseudoconvex boundary}

Let $\Omega \subseteq \mathbf{C}^{k}$ be a domain with $C^{2}$ smooth boundary b $\Omega$, and assume $\Omega$ is strongly pseudoconvex at $0 \in \mathrm{b} \Omega$. Then there is an open neighborhood $U$ of 0 in $\mathbf{C}^{k}$ and a $C^{2}$ smooth function $\varphi: U \rightarrow \mathbf{R}$ with the following properties:

$$
\begin{aligned}
& \Omega \cap U=\{z \in U: \varphi(z)<0\} \\
& \varphi(0)=0, \\
& \operatorname{grad} \varphi(0) \neq 0, \text { and } \\
& \text { the Levi form }\left(\partial^{2} \varphi(0) / \partial z_{i} \partial \bar{z}_{j}\right)_{1 \leqq i, j \leqq k} \text { is strictly } \\
& \text { positive definite. }
\end{aligned}
$$

After analytic change of coordinates in $\mathbf{C}^{k}$, we may assume $\varphi$ has the form

$$
\varphi(z)=\operatorname{Im} z_{k}+\sum_{j=1}^{k} c_{j}\left|z_{j}\right|^{2}+O\left(|z|^{3}\right),
$$

where $c_{j}>0$ is constant, $1 \leqq j \leqq k$. (A proof of this fact may be found in Hörmander, [4].)

Let $\mathscr{A}_{k}(\{n !\})$ be a proper subset of $\mathscr{A}_{k}\left(\left\{M_{n}\right\}\right), k \geqq 2$, a quasianalytic local algebra as defined in the Introduction. Set $z^{\prime}=\left(z_{1}, \ldots, z_{k-1}\right)$ and $f(z)=f\left(z^{\prime}, z_{k}\right)=$ $=z_{k}^{2}+z_{1}$. Then $f$ is an analytic Weierstrass polynomial of degree two in $z_{k}$. For $a \in \mathbf{C}$, set $g(z, a)=e^{i a z_{k}}$. Note that for each $a \in \mathbf{C}, g \in \mathcal{O}_{k} \subseteq \mathscr{A}_{k}\left(\left\{M_{n}\right\}\right)$. Suppose it were possible to write for each $a \in \mathbf{C}$

$$
g=f q+r_{1} z_{k}+r_{2},
$$

where $q=q(z, a) \in \mathscr{A}_{k}\left(\left\{M_{n}\right\}\right)$ and $r_{1}=r_{1}\left(z^{\prime}, a\right), r_{2}=r_{2}\left(z^{\prime}, a\right) \in \mathscr{A}_{k-1}\left(\left\{M_{n}\right\}\right)$. Since the roots of $f\left(z^{\prime}, z_{k}\right)=0$ are $z_{k}= \pm i \sqrt{z_{1}}$, it would follow from equation (3.2) that

$$
r_{1}\left(z^{\prime}, a\right)=i\left(e^{a \sqrt{z_{1}}}-e^{-a \sqrt{z_{1}}}\right) / 2 \sqrt{z_{1}} .
$$

Now consider only values of $a \in \mathbf{R}$ with $a<0$. If $z \in \bar{\Omega}$ and $|z|$ is sufficiently small, it follows from equation (3.1) that $\operatorname{Im} z_{k} \leqq 0$. Thus, if $\alpha \in\left(\mathbf{Z}^{+}\right)^{k},|\alpha| \leqq n \in \mathbf{Z}^{+}, z \in \bar{\Omega}$, and $|z|$ is sufficiently small, then we get

$$
\begin{aligned}
\left|D_{z}^{\alpha} g(z, a)\right| & \leqq|a|^{|\alpha|} e^{-a \operatorname{Im} z_{k}} \\
& \leqq|a|^{|\alpha|} \\
& \leqq\left(|a|^{|\alpha|} / M_{|\alpha|}\right) M_{|\alpha|} \\
& \leqq \lambda(a) M_{n} .
\end{aligned}
$$

Thus, for each $a<0, g=g(z, a) \in A_{k, 1,1}$. If we apply Lemma 2.2, it follows that there exist $\varepsilon_{1}>0$ and $A_{1}>0$, both independent of $a$, such that

$$
\sup _{z^{\prime} \in \bar{\Omega} \cap \Delta_{k-1}\left(\varepsilon_{1}\right)}\left|r_{1}\left(z^{\prime}, a\right)\right| \leqq A_{1} \lambda(a), \quad a<0 .
$$


Let $\varepsilon>0, z^{\prime}=(\varepsilon, 0, \ldots, 0)$, and $z_{k}=-i \varepsilon$. If $\varepsilon$ is chosen sufficiently small, then $z^{\prime} \in \Delta_{k-1}\left(\varepsilon_{1}\right)$ and $\varphi(z)=-\varepsilon+c_{1} \varepsilon^{2}+c_{k} \varepsilon^{2}+O\left(\varepsilon^{3}\right)<0$, so that $z \in \bar{\Omega}$. Thus estimate (3.4) yields

$$
\left|e^{a \sqrt{\varepsilon}}-e^{-a \sqrt{\varepsilon}}\right| / 2 \sqrt{\varepsilon} \leqq A_{1} \lambda(a), \quad a<0 .
$$

Since $\left(e^{a} \sqrt{e}-e^{-a \sqrt{\varepsilon}}\right) / 2 \sqrt{\varepsilon}$ is asymptotic to $e^{-a \sqrt{\varepsilon}} / 2 \sqrt{\varepsilon}$, inequality (3.5) implies there exist constants $A>0$ and $K>0$, both independent of $a$, such that

$$
e^{a \sqrt{\varepsilon}} \leqq K \lambda(a), \quad a>A .
$$

Inequality (3.6) together with Lemma 2.3 now imply the existence of constants $\alpha>0$. and $\beta>0$ such that

$$
M_{n} \leqq \alpha \beta^{n} n !, \quad n \in \mathbf{Z}^{+} .
$$

This implies $\mathscr{A}_{k}\left(\left\{M_{n}\right\}\right)=\mathscr{A}_{k}(\{n !\})$, contrary to assumption. We conclude the Weierstrass division theorem does not generalize to $\mathscr{A}_{k}\left(\left\{M_{n}\right\}\right)$ when $\mathscr{A}_{k}\left(\left\{M_{n}\right\}\right) \supsetneqq \mathscr{A}_{k}(\{n !\})$ and $k \geqq 2$. Indeed, we have shown that it isn't always possible to divide in $\mathscr{A}_{k}\left(\left\{M_{n}\right\}\right)$ by Weierstrass polynomials from $\mathcal{O}_{k-1}\left[z_{k}\right]$.

\section{The case of a pseudoflat boundary}

Let $\Omega \subseteq \mathbf{C}^{k}, k \geqq 2$, be a product domain with 0 a member of the pseudoflat part of $\mathrm{b} \Omega$. Thus, let $U_{1} \subseteq \mathbf{C}$ be any plane domain with $0 \in \mathrm{b} U_{1}$, let $U_{j}=$ $=\{z \in \mathbf{C}:|z|<1\}$ be the open unit disc in the plane for $2 \leqq j \leqq k$, and let $\Omega=U_{1} \times$ $\times U_{2} \times \ldots \times U_{k}$. Let $\mathscr{A}_{k}\left(\left\{M_{n}\right\}\right)$ be a quasianalytic local algebra. We show in this section that a generic division theorem holds in $\mathscr{A}_{k}\left(\left\{M_{n}\right\}\right)$. We also show that division is possible in $\mathscr{A}_{k}\left(\left\{M_{n}\right\}\right)$ by every regular element of $\mathcal{O}_{k}$.

By a generic monic polynomial in $z_{k}$ of degree $d$ we mean an element in $\mathbf{C}\left[z_{k}\right]$ of the form $P_{d}\left(z_{k}, \lambda\right)=z_{k}^{d}+\sum_{j=1}^{d} \lambda_{j} z_{k}^{d-j}$, where $\lambda=\left(\lambda_{1}, \ldots, \lambda_{d}\right) \in \mathbf{C}^{d}$.

Theorem 4.1. (Generic division theorem for $\mathscr{A}_{k}\left(\left\{M_{n}\right\}\right)$ ) Let $P_{d}=P_{d}\left(z_{k}, \lambda\right)$ be a generic polynomial in $z_{k}$ of degree $d$. For each $g \in \mathscr{A}_{k}\left(\left\{M_{n}\right\}\right)$, there exists $\varepsilon>0$ such that if $\lambda \in \Delta_{d}(\varepsilon)$, then there exist unique elements $q=q(z, \lambda) \in \mathscr{A}_{k}\left(\left\{M_{n}\right\}\right)$ and $r_{j}=r_{j}\left(z^{\prime}, \lambda\right) \in \mathscr{A}_{k-1}\left(\left\{M_{n}\right\}\right), 1 \leqq j \leqq d$, such that

$$
g=P_{d} q+\sum_{j=1}^{d} r_{j} z_{k}^{d-j} .
$$

Furthermore, all the germs in equation (4.1) are defined for $(z, \lambda) \in\left(\bar{\Omega} \cap \Delta_{k}(\varepsilon)\right) \times \Delta_{d}(\varepsilon)$ and are analytic in $(z, \lambda)$ on $\left(\Omega \cap \Delta_{k}(\varepsilon)\right) \times \Delta_{d}(\varepsilon)$.

Proof. Choose $0<r<1$ so that the germ $g$ is defined on $\bar{\Omega} \cap \Delta_{k}(r)$. Let $0<\delta<r$. By Cauchy's integral formula, if $z \in \bar{\Omega} \cap \Delta_{k}(\delta / 2)$, then

$$
g(z)=\frac{1}{2 \pi i} \int_{|\zeta|=\delta} \frac{g\left(z^{\prime}, \zeta\right)}{\zeta-z_{k}} d \zeta .
$$


Observe that

and so

$$
\begin{aligned}
P_{j}(\zeta, \lambda) & =\zeta^{j}+\sum_{i=1}^{j} \lambda_{i} \zeta^{j-i} \\
& =\zeta\left(\zeta^{j-1}+\sum_{i=1}^{j-1} \lambda_{i} \zeta^{j-1-i}\right)+\lambda_{j} \\
& =\zeta P_{j-1}(\zeta, \lambda)+\lambda_{j}
\end{aligned}
$$

Thus

$$
-\lambda_{j}=\zeta P_{j-1}(\zeta, \lambda)-P_{j}(\zeta, \lambda)
$$

$$
\begin{aligned}
& P_{d}(\zeta, \lambda)-P_{d}\left(z_{k}, \lambda\right) \\
= & \zeta P_{d-1}(\zeta, \lambda)+\lambda_{d}-\sum_{j=1}^{d} \lambda_{j} z_{k}^{d-j}-z_{k}^{d} \\
= & \zeta P_{d-1}(\zeta, \lambda)+\sum_{j=1}^{d-1}\left(-\lambda_{j}\right) z_{k}^{d-j}-z_{k}^{d} \\
= & \zeta P_{d-1}(\zeta, \lambda)+\sum_{j=1}^{d-1}\left[\zeta P_{j-1}(\zeta, \lambda)-P_{j}(\zeta, \lambda)\right] z_{k}^{d-j}-z_{k}^{d} \\
= & \sum_{j=1}^{d} \zeta P_{j-1}(\zeta, \lambda) z_{k}^{d-j}-\sum_{j=0}^{d-1} P_{j}(\zeta, \lambda) z_{k}^{d-j} \\
= & \sum_{j=1}^{d} \zeta P_{j-1}(\zeta, \lambda) z_{k}^{d-j}-\sum_{j=1}^{d} P_{j-1}(\zeta, \lambda) z_{k}^{d-j+1} \\
= & \left(\sum_{j=1}^{d} P_{j-1}(\zeta, \lambda) z_{k}^{d-j}\right)\left(\zeta-z_{k}\right) .
\end{aligned}
$$

Adding $P_{d}\left(z_{k}, \lambda\right)$ to both sides of the identity we have obtained, viz.,

$$
P_{d}(\zeta, \lambda)-P_{d}\left(z_{k}, \lambda\right)=\left(\sum_{j=1}^{d} P_{j-1}(\zeta, \lambda) z_{k}^{d-j}\right)\left(\zeta-z_{k}\right)
$$

and dividing through by $P_{d}(\zeta, \lambda)\left(\zeta-z_{k}\right)$, we obtain

$$
\frac{1}{\zeta-z_{k}}=\frac{P_{d}\left(z_{k}, \lambda\right)}{P_{d}(\zeta, \lambda)\left(\zeta-z_{k}\right)}+\sum_{j=1}^{d} \frac{P_{j-1}(\zeta, \lambda)}{P_{d}(\zeta, \lambda)} z_{k}^{d-j} .
$$

Now choose $s>0$ such that $\lambda \in \Delta_{d}(s)$ implies that the roots of $P_{d}\left(z_{k}, \lambda\right)$ are contained in $\Delta_{1}(\delta / 2)$. If $z \in \bar{\Omega} \cap \Delta_{k}(\delta / 2)$ and $\lambda \in \Delta_{d}(s)$, substitution of expression (4.3) for $1 /\left(\zeta-z_{k}\right)$ into equation (4.2) yields

$$
\begin{aligned}
g(z) & =\left[\frac{1}{2 \pi i} \int_{|\zeta|=\delta} \frac{g\left(z^{\prime}, \zeta\right)}{P_{d}(\zeta, \lambda)\left(\zeta-z_{k}\right)} d \zeta\right] P_{d}\left(z_{k}, \lambda\right) \\
& +\sum_{j=1}^{d}\left[\frac{1}{2 \pi i} \int_{|\zeta|=\delta} \frac{g\left(z^{\prime}, \zeta\right) P_{j-1}(\zeta, \lambda)}{P_{d}(\zeta, \lambda)} d \zeta\right] z_{k}^{d-j} .
\end{aligned}
$$


Thus, we get an equation of the form (4.1) with

$$
q(z, \lambda)=\frac{1}{2 \pi i} \int_{|\zeta|=\delta} \frac{g\left(z^{\prime}, \zeta\right)}{P_{d}(\zeta, \lambda)\left(\zeta-z_{k}\right)} d \zeta
$$

and

$$
r_{j}\left(z^{\prime}, \lambda\right)=\frac{1}{2 \pi i} \int_{|\zeta|=\delta} \frac{g\left(z^{\prime}, \zeta\right) P_{j-1}(\zeta, \lambda)}{P_{d}(\zeta, \lambda)} d \zeta, \quad 1 \leqq j \leqq d .
$$

Let $\varepsilon=\min (\delta / 2, s)$, and note $\left|P_{d}(\zeta, \lambda)\right| \geqq C>0$ and $\left|\zeta-z_{k}\right| \geqq \varepsilon>0$ for $|\zeta|=\delta$ and $\lambda \in \Delta_{d}(\varepsilon)$. We may thus differentiate under the integral sign in equation (4.4) and obtain that $q(z, \lambda)$ is analytic in $(z, \lambda)$ on $\left(\Omega \cap \Delta_{k}(\varepsilon)\right) \times \Delta_{d}(\varepsilon)$. Also, since $g(z)$ represents an element of $\mathscr{A}_{k}\left(\left\{M_{n}\right\}\right)$ on $\bar{\Omega} \cap \Delta_{k}(\delta)$, there exist $A_{1}>0$ and $B_{1}>0$ such that for all $n \in \mathbf{Z}^{+}$,

Thus,

$$
\sup _{\substack{\alpha \in\left(Z^{+}\right)^{k},|\alpha| \leqq n, z \in \bar{\Omega} \cap \Delta_{k}(\delta)}}\left|D^{\alpha} g(z)\right| \leqq A_{I} B_{1}^{n} M_{n} .
$$

$$
\sup _{\substack{\alpha \in\left(\mathbf{Z}^{+}\right)^{k-1},|\alpha| \leqq n,\left(z^{\prime}, \zeta\right) \in \bar{\Omega} \Lambda_{k}(\delta)}}\left|D_{z^{\prime}}^{\alpha} g\left(z^{\prime}, \zeta\right)\right| \leqq A_{1} B_{1}^{n} M_{n} .
$$

Since $\left|P_{d}(\zeta, \lambda)\right|$ and $\left|\zeta-z_{k}\right|$ are bounded away from 0 for $|\zeta|=\delta$ and $(z, \lambda) \epsilon$ $\epsilon\left(\bar{\Omega} \cap \Delta_{k}(\varepsilon)\right) \times \Delta_{d}(\varepsilon)$, it follows that there exist $A>0$ and $B>0$, both independent of $\zeta$ with $|\zeta|=\delta$ and $\lambda \in \Delta_{d}(\varepsilon)$, such that

$$
\sup _{\substack{\alpha \in\left(\mathbb{Z}^{+}\right)^{k},|\alpha| \leqq n, z \in \bar{\Omega} \cap \Delta_{k}(\varepsilon)}}\left|D_{z}^{\alpha}\left[g\left(z^{\prime}, \zeta\right) / P_{d}(\zeta, \lambda)\left(\zeta-z_{k}\right)\right]\right| \leqq A B^{n} M_{n} .
$$

We may thus differentiate under the integral sign in equation (4.4), estimate in a straightforward manner, and obtain that if $\lambda \in \Delta_{d}(\varepsilon)$, then $q=q(z, \lambda)$ represents an element of $\mathscr{A}_{k}\left(\left\{M_{n}\right\}\right)$ on $\bar{\Omega} \cap \Delta_{k}(\varepsilon)$. A similar argument shows that if $\lambda \in \Delta_{d}(\varepsilon)$, then $r_{j}=r_{j}\left(z^{\prime}, \lambda\right)$ in equation (4.5) represents an element of $\mathscr{A}_{k-1}\left(\left\{M_{n}\right\}\right)$ on $\bar{\Omega} \cap \Delta_{k-1}(\varepsilon)$ which is analytic in $\left(z^{\prime}, \lambda\right)$ on $\left(\Omega \cap \Delta_{k-1}(\varepsilon)\right) \times \Delta_{d}(\varepsilon)$ for $1 \leqq j \leqq d$.

Finally, to prove uniqueness, suppose that

$$
\begin{aligned}
g & =P_{d} q+\sum_{j=1}^{d} r_{j} z_{k}^{d-j} \\
& =P_{d} \tilde{q}+\sum_{j=1}^{d} \tilde{r}_{j} z_{k}^{d-j},
\end{aligned}
$$

where $q=q(z, \lambda), \tilde{q}=\tilde{q}(z, \lambda) \in \mathscr{A}_{k}\left(\left\{M_{n}\right\}\right)$ and $r_{j}=r_{j}\left(z^{\prime}, \lambda\right), \tilde{r}_{j}=\tilde{r}_{j}\left(z^{\prime}, \lambda\right) \in \mathscr{A}_{k-1}\left(\left\{M_{n}\right\}\right)$ for $1 \leqq j \leqq d$ and for each $\lambda \in \mathbf{C}^{d}$ which is sufficiently small. Then for some $\varepsilon>0$ and all $(z, \lambda) \in\left(\bar{\Omega} \cap \Delta_{k}(\varepsilon)\right) \times \Delta_{d}(\varepsilon)$,

$$
\sum_{j=1}^{d}\left(r_{j}\left(z^{\prime}, \lambda\right)-\tilde{r}_{j}\left(z^{\prime}, \lambda\right)\right) z_{k}^{d-j}=P_{d}\left(z_{k}, \lambda\right)(\tilde{q}(z, \lambda)-q(z, \lambda)) .
$$


$P_{d}\left(z_{k}, \lambda\right)$ has exactly $d$ zeros, while $\sum_{j=1}^{d}\left(r_{j}\left(z^{\prime}, \lambda\right)-\tilde{r}_{j}\left(z^{\prime}, \lambda\right)\right) z_{k}^{d-j}$ is a polynomial in $z_{k}$ of degree at most $d-1$. Thus $r_{j}=\tilde{r}_{j}$ for $1 \leqq j \leqq d$ and $q=\tilde{q}$.

Theorem 4.2. Let $f=f(z) \in \mathcal{O}_{k}, k \geqq 2$, be regular in $z_{k}$ of order $d$. Then we may divide by $f$ in $\mathscr{A}_{k}\left(\left\{M_{n}\right\}\right)$.

Proof. Since $f \in \mathcal{O}_{k}$ is regular in $z_{k}$ of order $d$, we may apply the Weierstrass preparation theorem in $\mathcal{O}_{k}$ to write

$$
f=u P,
$$

where $u \in \mathcal{O}_{k}$ is a unit and $P \in \mathcal{O}_{k-1}\left[z_{k}\right]$ is a Weierstrass polynomial in $z_{k}$ of degree $d$. Let $g \in \mathscr{A}_{k}\left(\left\{M_{n}\right\}\right)$. If we can perform the division

$$
g=P q^{\prime}+r^{\prime},
$$

where $q^{\prime} \in \mathscr{A}_{k}\left(\left\{M_{n}\right\}\right)$ and $r^{\prime} \in \mathscr{A}_{k-1}\left(\left\{M_{n}\right\}\right)\left[z_{k}\right]$, then we can obtain the division

$$
g=f q+r
$$

by taking $q=u^{-1} q^{\prime} \in \mathscr{A}_{k}\left(\left\{M_{n}\right\}\right)$ and $r=r^{\prime} \in \mathscr{A}_{k-1}\left(\left\{M_{n}\right\}\right)\left[z_{k}\right]$. Thus, we may assume $f=P$.

Choose a polydisc $\Delta_{k}(r)$ such that the germ $P$ is defined on $\Delta_{k}(r)$ and the germ $g$ is defined for $z \in \bar{\Omega} \cap \Delta_{k}(r)$. Since $P$ is a Weierstrass polynomial in $z_{k}$, we can find numbers $\delta_{j}$ with $0<\delta_{j}<r, 1 \leqq j \leqq k$, such that $P(z) \neq 0$ if $\left|z_{k}\right|=\delta_{k}$ and $\left|z_{j}\right| \leqq \delta_{j}$, $1 \leqq j \leqq k-1$. Let $\Delta_{k}(\delta)=\left\{z \in \mathbf{C}^{k}:\left|z_{j}\right|<\delta_{j}\right.$ for $\left.1 \leqq j \leqq k\right\}$. For $z \in \bar{\Omega} \cap \Delta_{k}(\delta)$, define

$$
q(z)=\frac{1}{2 \pi i} \int_{|\zeta|=\delta_{k}} \frac{g\left(z^{\prime}, \zeta\right)}{P\left(z^{\prime}, \zeta\right)} \frac{d \zeta}{\zeta-z_{k}}
$$

and

$$
r(z)=\frac{1}{2 \pi i} \int_{|\zeta|=\delta_{k}} \frac{g\left(z^{\prime}, \zeta\right)}{P\left(z^{\prime}, \zeta\right)} \frac{P\left(z^{\prime}, \zeta\right)-P\left(z^{\prime}, z_{k}\right)}{\zeta-z_{k}} d \zeta .
$$

By the Cauchy integral theorem, if $z \in \Omega \cap \Delta_{k}(\delta)$, then

$$
\begin{aligned}
P(z) q(z)+r(z) & =\frac{1}{2 \pi i} \int_{|\zeta|=\delta_{k}} \frac{g\left(z^{\prime}, \zeta\right)}{\zeta-z_{k}} d \zeta \\
& =g(z) .
\end{aligned}
$$

Since $P$ is a Weierstrass polynomial in $z_{k}$ of degree $d, r$ is a polynomial in $z_{k}$ of degree at most $d-1$. We may differentiate under the integral signs in equations (4.6) and (4.7) and see that $q$ and $r$ are analytic in $z$ on $\Omega \cap \Delta_{k}(\delta)$. Since $g$ represents an element of $\mathscr{A}_{k}\left(\left\{M_{n}\right\}\right)$ on $\bar{\Omega} \cap \Delta_{k}(\delta)$, there exist $A_{1}>0$ and $B_{1}>0$ such that for all $n \in \mathbf{Z}^{+}$,

$$
\sup _{\substack{\alpha \in\left(\mathbf{Z}^{+}\right)^{k},|\alpha| \leqq n, ~ \\ z \in \bar{\Omega} \cap \Delta_{k}(\delta)}}\left|D^{\alpha} g(z)\right| \leqq A_{1} B_{1}^{n} M_{n}
$$


Thus for all $\zeta$ with $|\zeta|=\delta_{k}$ and all $n \in \mathbb{Z}^{+}$,

$$
\sup _{\substack{\alpha \in\left(\mathrm{Z}^{+} \boldsymbol{j}^{k-1},|\alpha| \leqq n, z^{\prime} \in \bar{\Omega} \cap A_{k-1}(\delta)\right.}}\left|D_{z^{\prime}}^{\alpha} g\left(z^{\prime}, \zeta\right)\right| \leqq A_{1} B_{1}^{n} M_{n} .
$$

Since $P\left(z^{\prime}, \zeta\right) \neq 0$ for $|\zeta|=\delta_{k}$ and $\left|z_{j}\right|<\delta_{j}, \quad 1 \leqq j \leqq k-1, \quad\left|P\left(z^{\prime}, \zeta\right)\right| \geqq C>0$ for $|\zeta|=\delta_{k}$ and $\left|z_{j}\right|<\delta_{j} / 2,1 \leqq j \leqq k-1$. Also $\left|\zeta-z_{k}\right| \geqq \delta_{k} / 2$ for $|\zeta|=\delta_{k}$ and $\left|z_{k}\right|<$ $<\delta_{k} / 2$. Let $\varepsilon=\min _{1 \leqq j \leqq k} \delta_{j} / 2$. It follows that there exist $A>0$ and $B>0$, both independent of $\zeta$ with $|\zeta|=\delta_{k}$, such that for all $n \in \mathbf{Z}^{+}$,

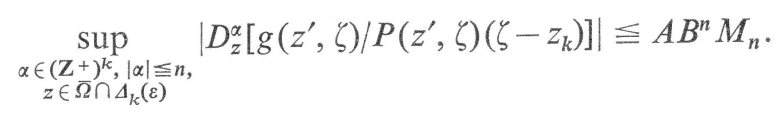

We may now differentiate under the integral sign in equation (4.6) and estimate in a straightforward manner to see that $g$ represents an element of $\mathscr{A}_{k}\left(\left\{M_{n}\right\}\right)$ on $\bar{\Omega} \cap \Delta_{k}(\varepsilon)$. A similar argument shows that $r$, given by equation (4.7), represents an element of $\mathscr{A}_{k-1}\left(\left\{M_{n}\right\}\right)\left[z_{k}\right]$ on $\bar{\Omega} \cap \Delta_{k}(\varepsilon)$.

To prove uniqueness, suppose

$$
g=P q+r=P \tilde{q}+\tilde{r} .
$$

Then for some polydisc $\Delta_{k}$ and all $z \in \bar{\Omega} \cap \Delta_{k}$,

$$
r(z)-\tilde{r}(z)=P(z)(\tilde{q}(z)-q(z)) .
$$

For $z^{\prime}$ sufficiently small, $P\left(z^{\prime}, z_{k}\right)$ has exactly $d$ zeros, while $r(z)-\tilde{r}(z)$ is a polynomial in $z_{k}$ of degree at most $d-1$. Hence $\tilde{r}=r$ and $\tilde{q}=q$.

Corollary 4.3. Let $k \geqq 2, P \in \mathcal{O}_{k-1}\left[z_{k}\right]$ be a Weierstrass polynomial, and $f \in \mathscr{A}_{k}\left(\left\{M_{n}\right\}\right)$. If $f P \in \mathscr{A}_{k-1}\left(\left\{M_{n}\right\}\right)\left[z_{k}\right]$ is a polynomial, then $f$ is a polynomial, $f \in \mathscr{A}_{k-1}\left(\left\{M_{n}\right\}\right)\left[z_{k}\right]$.

Proof. Since $f P$ and $P$ are polynomials in $z_{k}$ over $\mathscr{A}_{k-1}\left(\left\{M_{n}\right\}\right)$, we may apply the algebraic division theorem for polynomials to write

$$
f P=P q+r,
$$

where $q, r \in \mathscr{A}_{k-1}\left(\left\{M_{n}\right\}\right)\left[z_{k}\right]$ and $r$ has degree less than the degree of $P$. By the uniqueness part of Theorem 4.2, $\quad r=0$ and $f=q$. Thus $f \in \mathscr{A}_{k-1}\left(\left\{M_{n}\right\}\right)\left[z_{k}\right]$, as desired.

In closing, we mention one final application of Theorem 4.2. Let $R$ be a commutative ring with unit and $M$ be an $R$-module. $M$ is a flat $R$-module if for every exact sequence of $R$-modules $A \rightarrow B \rightarrow C$, the tensored sequence

$$
A \underset{R}{\otimes} M \rightarrow B \underset{R}{\otimes} M \rightarrow C \underset{R}{\otimes} M
$$

is also exact. It is possible to use Theorem 4.2 to establish that $\mathscr{A}_{k}\left(\left\{M_{n}\right\}\right)$ is a flat ring extension of $\mathcal{O}_{k}, k \geqq 2$. The details are so similar to those found in Nagel [7], however, that we choose to omit them. 


\title{
References
}

[1] Atryah, M. F., and I. G. MacDonald: Introduction to commutative algebra. - AddisonWesley Publishing Co., Inc., Reading, Mass. 1969.

[2] Childress, C. L.: Weierstrass division in quasianalytic local rings. - To appear.

[3] Grothendieck, A.: Espaces vectoriels topologiques. - Sociedade de Mathemática de São Paulo, Sãn Paulo, 1964.

[4] Hörmander, L.: An introduction to complex analysis in several variables. - North-Holland Publishing Company, 1973.

[5] Mather, J. N.: On Nirenberg's proof of Malgrange's preparation theorem. - Proceedings of Liverpool Singularities-Symposium I, ed. C.T.C. Wall, Springer-Verlag, Berlin, 1971.

[6] Mather, J. N.: Stability of $C^{\infty}$ mappings. I. The division theorem. - Ann. of Math. (2) 87, $1968,89-104$.

[7] Nagel, A.: Flatness criteria for modules of holomorphic functions over $\mathcal{O}_{n}$. - Duke Math. J. 40, 1973, 433-448.

[8] Rudin, W.: Real and complex analysis. - McGraw-Hill Co., Inc., New York, 1966.

[9] WALL, C. T. C.: Introduction to the preparation theorem. - Proceedings of Liverpool Singularities - Symposiom I, ed. C. T. C. Wall, Springer-Verlag, Berlin-HeidelbergNew York, 1971.

\author{
Case Western Reserve University \\ Department of Mathematics and Statistics \\ Cleveland, Ohio 44106 \\ USA
}

Received 26 January 1976 